\title{
CONSTRAINT INDUCED MOVEMENT THERAPY AND REAL-WORLD ARM USE IN CHILDREN WITH HEMIPARESIS
}

\author{
Asmaa Osama Sayed *1, Hebatallah Mohamed Kamal ${ }^{1}$, Nagham Mohamed Kamal \\ Khalil ${ }^{2}$.
}

${ }^{1}$ Department of Physical Therapy for Growth and Developmental Disorders in Children and It's Surgery, Faculty of Physical Therapy, Cairo University, Egypt.

${ }^{2}$ Physical therapy department at El-Raml Children Hospital,Master degree in physical therapy, Cairo University, Egypt.

\section{ABSTRACT}

Introduction: Constraint Induced Movement therapy is a rehabilitative technique used in physical rehabilitation that restrains the sound arm to improve the functional ability of the affected upper extremity. This study was conducted to explain the effect of constraint induced movement therapy on functional use of spastic arm in hemiparetic cerebral palsied children.

Materials and Methods: The study was conducted on thirty children with spastic hemiparesis of both sexes (seventeen boys and thirteen girls); there age ranged between 2 to 6 years old. They were classified randomly into two equal groups (A \& B). Group (A): received co-nstraint induced movement therapy in addition to selected occupational therapy for 3 months, every day with session duration two hours. Group (B): received only traditional occupational therapy, session duration two hours. Assessment of real-world arm movement (reaching and grasping) was done by pediatric motor activity log-revised (PMAL-R) before and after 3 months of treatment. Results: Study revealed statistically significant improvement in real-world arm movement in favor to group (A), regarding to hand dominancy the P-value was Pre $(0.540)$ and Post $\left(<0.001^{*}\right)$.The percentage of change in group (A) (Pre and Post training) was (116.79) and in group (B) was (37.50)g. We can conclude that constraint induced movement therapy improved real-world arm movement in spastic hemiparetic cerebral palsied children.

KEY WORDS:Cerebral palsy, Hemiparesis, Spasticity, Constraint Induced Movement Therapy, Pediatric motor activity Log-Revised, Reaching and Grasping.

Address for correspondence: Dr. Asmaa Osama Sayed, Lecturer, Department of Physical Therapy for Growth and Developmental Disorders in Children and It's Surgery, Faculty of Physical Therapy, Cairo University, Egypt. E-Mail: drasmaaosama@yahoo.com

\begin{tabular}{|c|c|c|}
\hline \multicolumn{3}{|c|}{ Access this Article online } \\
\hline Quick Response code & \multicolumn{2}{|c|}{$\begin{array}{l}\text { International Journal of Physiotherapy and Research } \\
\qquad \text { ISSN } 2321-1822 \\
\text { www.ijmhr.org/ijpr.html }\end{array}$} \\
\hline DoI: $10.16965 /$ ijpr.2017.149 & $\begin{array}{l}\text { Received: } 27-03-2017 \\
\text { Peer Review: 27-03-2017 } \\
\text { Revised: } 25-04-2017\end{array}$ & $\begin{array}{l}\text { Accepted: 13-05-2017 } \\
\text { Published (O): 11-06-2017 } \\
\text { Published (P): 11-06-2017 }\end{array}$ \\
\hline
\end{tabular}

\section{INTRODUCTION}

Cerebral palsy is defined as a group of movement and posture disorders caused by a nonprogressive but permanent abnormality in the fetal or infant brain [1]. Cerebral palsy occurs in about two of every thousand live births, and with improving neonatal care and premature birth survival rates these numbers may increase
[2]. Increased muscle tone results in limited movement, especially in the arms and legs. Spastic CP is the most common type, affecting approximately $70 \%$ of all cases with CP. This form is caused predominantly due to damage to the motor cortex and/or pyramidal tract, Hemiplegia is included within spastic type [3] In hemiplegia, one side of the body is more impaired than 
the other; the upper limb is typically more affected than the lower limb [4]. The Modified Ashworth Scale (MAS) is the most widely used measure of spasticity. It is simple to perform and does not require special equipment or extensive training. It measures only one aspect of spasticity and it's administration takes less than 5 minutes [5]. Children with hemiplegic CP often compensate for their weak arm by choosing to perform most tasks with their unaffected arm this leaded to developmental disregard or learned non-use where the affected limb is further inhibited from healthy development [6]. Constraint induced movement therapy(CIMT) is used in children with spastic hemiparesis [7]. Some studies have yielded positive outcomes for all participants who receive CIMT [8]. Other studies have shown that more research is needed to confirm the most effective way of implementing the intervention in order to maximize the therapeutic benefits for participants [9]. shaping and repetitive task practice during a six hour program used for adults may be too disturbing for children. In order to make the intervention child-friendly, both structured-practice tasks and the type of constraint must be adapted to suit children [10]. Motor training focused on activities rather than on the individual movements required to complete activities, which had shown to greatly enhance gains in spontaneous use of the more-affected upper extremity [11].

In Constraint induced movement therapy efficacy studies recommended that Pediatric Motor Activity Log-Revised scale (PMAL-R) administered before and after treatment and weekly for a month following treatment [12]. Parents were asked to rate how well (HW) and how often $(\mathrm{HO})$ their children use their moreaffected arm to accomplish each activity over a specified period. Both scales, which range from $0-5$, are anchored at six points by abbreviated phrases and longer definitions; interviewees may select scores halfway between the anchors. Conversion for $\mathrm{HW}$ items scores $(0,1$, or $2=0 ; 3$ $=1 ; 4$ or $5=2$ ) and $\mathrm{HO}$ item scores ( 0 or $1=0 ; 2$ or $3=1 ; 4$ or $5=2$ ) based on the mean of the item scores used to derive the total scores for each scale [13].

The PMAL-R had high internal consistency and
Convergent validity was supported by a strong correlation between changes in PMAL-R scores and more-affected arm use during play which verify The PMAL-R interview as a reliable and valid measure of upper-extremity pediatric neurorehabilitation [14]. Manual Ability Classification System (MACS) is designed to classify how children with $\mathrm{CP}$ use their hands when handling objects in daily activities. Such focus on manual ability follows the International Classification of Functioning, Disability and Health [15]. In addition to it reports the collaboration of both hands together: it is not an assessment of each hand separately. It looks for the children's manual ability to 'handle object(s) in daily life that are relevant and age appropriate for the child [16].

\section{MATERIALS AND METHODS}

\section{Participants, Randomization and Informed}

Consent: Thirty Cerebral Palsied children with Spastic Hemiparesis from both sexes participated in this study. All participants provided signed informed consent before randomization. Children were assigned randomly using the so called envelope method into the two groups. Letters A (study) and B (control) were written on 30 pieces of folded papers and each one was placed inside an envelope so the randomization process included asking children to select one folded paper to determine their group.

Design of the study: Group (A): 15 children participated in selected occupational therapy, CIMT and physical therapy for upper limb. Children were divided into two sub groups according to age of development into "pre-school" (2-4 year old) and "younger children" (4-6 year old).Group (B): 15children participated in selected occupational therapy only. The children were selected according to the following criteria: Inclusion criteria: Children age ranged from 2 to 6 years. Modified Ashworth Scale grade +1 or 2 (Spasticity level mild to moderate), Children with cerebral palsy spastic hemiparesis level MACS level II and Children were able to follow instructions during testing and treatment procedures. Exclusion criteria: Children with visual or hearing defects, Children with neurological or cognition disorders and Children with previous thoracic surgery. 


\section{Measures}

\section{Assessment materials:}

Materials of subject's selection: Modified Ashworth Scale (MAS), Manual Ability Classification Scale (MACS) level II- Handled most objects but with reduced quality and/or speed of achievement (independent in daily activities).

Materials for evaluation: Pediatric Motor Activity Log Revised (PMAL-R):_It provides functional assessment of functional use of the affected arm in normal life.It assesses 22 items; Eat finger foods. Pick up a small item. Self-feed with a fork/spoon. Brush teeth 5- Gesture Push arm through sleeve of clothing. Turn a page in a book. Point to a picture. Reach for an object above head. Push a button or key. Steady self. Open a door or cabinet. Turn a knob. Use arm to move across the floor 15- Take off shoes .Take off socks. Push a large object across the floor. Hold a ball Throw a ball or other object .Use a cylindrical object. Hold a handle while riding, pulling, or pushing a toy. Placement of an object. PMAL-R was applied to provide assessment pre and post treatment to assess the quality and quantity of motion in affected arm through how well and how often scales. The caregivers were asked about the actual movements that their children were able to do and this was initial evaluation which was checked by the evaluation done pre and post treatment. Children were asked to carry out 22 items first with the affected arm then with sound arm then comparison between both arms was done. The caregivers were asked to observe their children's activity in home and were given home routine for affected arm. The how well scale was carried out weekly.

Materials for treatment: 1- Constraint Induced Movement therapy: using slings to restrict the unaffected arm while still allowing for the arm to be used in the event of a trip or fall. It was an intensive therapy that required 2 hours per session, every day for 3 months and each task took 15 to 20 minutes.2- Selected Occupational Therapy program. 3- Selected Physical Therapy program.

Interventions: Constraint induced movement therapy is a therapeutic technique that consists of two main components: (1) Intensive training of the more-affected arm (2)Restraint of the less-affected arm, thereby forcing use of the more-affected arm [17]. There are 2 additional components:(3) Training by the behavioral technique termed "shaping" that involve(a) providing verbal feedback and verbal reward for small improvements in task performance;(b) selecting tasks that were tailored to address the motor deficits of the individual patient; (c) carry out parts of a movement sequence, if they were incapable of completing the movement on their own at first; and (d) systematically increasing the difficulty of the task performed' (4) Use of a "transfer package" of techniques to facilitate transfer of treatment gains from the clinic to everyday life [18].

Assessment of functional ability by PMAL-R "Rating Anchor":[0] Not used - Your child did not use the weaker arm for the activity.[1] Very poor - Your child had very little functional use of the weaker arm for the activity. The arm may have moved during the activity but was of no real functional help. [2] Poor - Your child had minor functional use of the weaker arm for the activity. The arm actively participated in the activity, but the stronger arm/caregiver did most of the work. [3] Fair or moderate - The weaker arm was used to accomplish the activity, but the performance was very slow and/or involved great difficulty. [4] Almost normal - The weaker arm was able to accomplish the activity independently but did so with some difficulty and/ or inaccuracy. [5] Normal-The weaker arm did the activity normally. The selected occupational therapy exercises: Playing with cubes. Putting cinctures in order (removing them and putting them back) Grasping cup for drinking. Pushing heavy weight .Rotating shoulder wheel .Using scissors to cut paper. Eating food using their hands .Eating using a fork Brushing teeth Waving their hands (good bye) Turning a page in a book. Holding a ball and throwing it. $\mathrm{He} /$ she should Place the object given to him/her appropriately. Home program was given to parents and caregivers: 1-Make children wear the sling for 1 hour at home, during this hour they should apply several activities with their affected arm.2-Observe their children throughout activities of the day and if they are able to generalize movement they gained through prac- 
tice in their daily living activities.

Data Analysis: Data were fed to the computer and analyzed using IBM SPSS software package version 20.0. Quantitative data were described using range (minimum and maximum), mean and standard deviation. The distributions of quantitative variables were tested for normality using Kolmogorov-Smirnov test, Shapiro-Wilk test and D'Agstino test. If it reveals normal data distribution, parametric tests was applied. If the data were abnormally distributed, non-parametric tests were used. For normally distributed data, comparison between two independent populations were done using independent t-test, also paired t-test is used to analyse two paired data. For abnormally distributed data, comparison between two independent populations were done using Mann Whitney test. Significance of the obtained results was judged at the $5 \%$ level.P value $\leq 0.05$ was considered significant.

\section{RESULT}

The age of both groups ranged from two to six years with a mean age ( \pm SD) of $4.15 \pm 1.19$ of group (A) and $4.07 \pm 1.26$ of group (B). There was no statistical significant difference between mean value of age of with $t$ value $=0.194$ and $p$ value $=0.848$. Both sexes were involved, six girls and nine boys in group $(A)$ and seven girls and eight boys in group (B). There is no statistical difference (matched), as regards gender distribution among both groups, 6 girls (40.0\%) and 9 boys $(60.0 \%)$ were present in group $(A)$ while 7 girls (46.7\%) and 8 boys (53.3\%) were present in group (B). (Chi square test $=0.136 ; p=0.713$ )।First comparison between each group was carried out using PMAL-R Pre training that reviled no significant difference between two groups using paired t-test with the P-value $(0.289)$ followed by comparison between each group Post training that reviled significant difference between two groups with the P-value $\left(<0.001^{*}\right)$.IISecondly comparison between both groups was carried out Pre and Post training using PMAL-R where it reviled significant difference between each group with P-value; Pre (0.289) and Post $\left(<0.001^{*}\right)$. In addition to Mann Whitney test represented by $Z$-value where it was $\left(Z=4.294^{*}\right)$ showing the percentage of change between each group pre and post training was (108.29) in group (A) and (31.08) in group (B).

Table 1: Comparison between the two studied groups according to PMAL-R.

\begin{tabular}{|c|c|c|c|c|}
\hline PMAL-R & Group A $(\mathbf{n}=15)$ & Group B $(\mathbf{n}=15)$ & Test of sig. & $P$ \\
\hline Pre & & & & \\
\hline Min. - Max. & $1.50-3.80$ & $1.87-3.30$ & & \\
\hline Mean \pm SD. & $2.76 \pm 0.73$ & $2.51 \pm 0.51$ & $\mathrm{t}=1.082$ & 0.289 \\
\hline & & & & \\
\hline Post & & & & \\
\hline Min. - Max. & $3.80-7.30$ & $1.90-4.50$ & & \\
\hline Mean \pm SD. & $5.57 \pm 1.19$ & $3.26 \pm 0.76$ & $\mathrm{t}=6.339^{*}$ & $<0.001^{*}$ \\
\hline & & & & \\
\hline \% of change & 108.29 & 31.08 & $\mathrm{Z}=4.294^{*}$ & $<0.001^{*}$ \\
\hline $\mathbf{p}_{1}$ & $<0.001^{*}$ & $<0.001^{*}$ & & \\
\hline
\end{tabular}

t: Student t-test Z: Z for Mann Whitney test

$p_{1}: p$ value for Paired $t$-test for comparing between pre and post in each group

*: Statistically significant at $\mathrm{p} \leq 0.05$.

\section{DISCUSSION}

This study mainly focused on the functional ability of children with hemiparesis cerebral palsy and the actual movements that they could perform alone without external help. That why The PMAL-R was chosen as it made it easier to assess actual functional ability of the child in several aspects involved in ADL before and after CIMT. Constraint induced movement therapy allowed the intensive use of the affected upper limb leading to enabling children to act independently throughout ADL. The results of this study showed positive effect of CIMT in improving arm hand function regarding daily use. The use of transfer package in CIMT showed positive effect regarding Changes From Pretreatment to 1 Year After Treatment As may be seen from the Data Supplement by telephone calls only, there was no decrease in PMAL-R Thus, the pattern of findings with respect to real-world outcome was the same at 1-year follow-up as at post-treatment.

The chosen age in this study was parallel to age in study of who reported measuring the moreaffected arm activity in children with upper-extremity hemiparesis measured by PMAL-R [19].

Appling CIMT was justified by who reported that children with unilateral CP typically learn strategies and techniques to perform daily tasks with just the unaffected hand and they typically did 
not use their affected upper extremity. Children frequently used only their unaffected upper extremity to increase the efficiency of performing tasks, which is known as developmental disregard. These problems further limited daily activities and participation of children with unilateral CP [7].Constraint induced movement therapy was the choice of treatment in present study as it constrained the unaffected upper extremity which prevented children from performing daily tasks with unaffected upper extremity leading to developmental disregard instead CIMT forced children with hemiparesis to perform ADL with affected extremity where the results showed positive improvement in functional arm hand movement which came in agreement with the study of that described CIMT as an efficacious in producing improvements in use of a more-affected upper extremity after central nervous system damage in adults. Using the same basic method, more improvements have been demonstrated in young children with CP [20].

Moreover the choice of intervention come in agreement with who stated that CIMT was an effective approach for treating developmental disregard in patients with unilateral motor involvement [21].

In addition to, the present study cope with several systematic reviews and randomized controlled trials (RCTs) had demonstrated that CIMT improved functional performance and ADL in children with CP which came in agreement with the results of the present study that showed significant difference in scores of PMAL-R post training in favor to group $(A)$ reflecting improvement in functional upper limb movement $[9,22]$.

Evaluation was conducted by The PMAL-R to assess the component of the transfer package in CIMT that could facilitate turning the therapeutic gains achieved in the clinic into real-world activities and prevent retention of the treatment effects 6 months later that was reported in previous studies which came in agreement with results of [23].

\section{CONCLUSION}

Based on the scope and findings of this study we conclude that Constraint induced movement therapy was found to be beneficial for real-world arm movement improvement in the affected upper limb of children with hemiparesis cerebral palsy.

\section{ABBREVIATIONS}

CIMT - Constraint Induced Movement Therapy

CP - Cerebral Palsy

HO - How Often

HW - How Well

MACS - Manual Ability Classification System

MAS - Modified Ashworth Scale

PMAL-R - Pediatric Motor Activity Log Revised

Conflicts of interest: None

\section{REFERENCES}

[1]. Bax M., Tydeman C., \& Flodmark O. Clinical and MRI correlates of cerebral palsy - The European cerebral palsy study. Journal of the American Medical Association, 2006;296(13):1602-1608.

[2]. Dong V, Tung I, Siu H, and Fong K. Studies comparing the efficacy of constraintinduced movement therapy and bimanual training in children with unilateral cerebral palsy: A systematic review. Developmental Neurorehabilitation (Informa UK Ltd.), 2012: 1-11.

[3]. Miller R, Hale L. Constraint-induced movement therapy for a youth with a chronic traumatic brain injury. NZ J Physiother, 2005;33:85-90.

[4]. Winckel $A$, et al. How does brain activation differ in children with unilateral cerebral palsy compared to typically developing children, during active and passive movements, and tactile stimulation? An fMRI study. Elsevier (Research in Developmental Disabilities), 2013;34:183-197.

[5]. Craven B, Morris A. Modified Ashworth scale reliability for measurement of lower extremity spasticity among patients with SCl. Spinal Cord. 2010;48 (3):207-13.

[6]. Hoare B., Wasia k., Imms C., and Carey L. Constraintinduced movement therapy in the treatment of the upper limb in children with hemiplegic cerebral palsy (Review).". The Cochrane Collaboration (John Wiley \& Sons, Ltd.), no. 1, 2009.

[7]. Hoare B, Imms C, Carey L, Wasiak J. Constraint-induced movement therapy in the treatment of the upper limb in children with hemiplegic cerebral palsy: a Cochrane systematic review. Clin Rehabil. 2007;21:675-685.

[8]. Al-Oraibi S., \& Eliasson A. C.. Implementation of constraint-induced movement therapy for young children with unilateral cerebral palsy in Jordan: A home-based model. Disability \& Rehabilitation, 2011;33(21-22), 2006-2012.

[9]. Huang H, Fetters L, Hale J, and McBride A. Bound for success: a systematic review of constraint-induced movement therapy in children with cerebral palsy supports improved arm and hand use. Phys Ther 2009;89:1126-41. 
[10]. Gordon A, Charles J, Wolf S. Methods of constraint induced movement therapy for children with hemiplegic cerebral palsy: development of a childfriendly intervention for improving upper-extremity function. Arch Phys Med Rehabil. 2005; 86:837844.

[11]. Gauthier L, Taub E, Perkins C, et al .Remodeling the brain: plastic structural brain changes produced by different motor therapies after stroke. Stroke 2008; 39:1520-1525.

[12]. Taub E., Ramey S., Deluca S., \& Echols K. Efficacy of constraint-induced movement therapy for children with cerebral palsy with asymmetric motor impairment. Pediatrics, 2004;113(2):305-312.

[13]. Wallen M, Bundy A, Pont K, Ziviani J. Psychometric properties of the Pediatric Motor Activity Log used for children with cerebral palsy. Dev Med Child Neurol. 2009;51:200-8.

[14]. Uswatte G, Taub E, Griffin A, Vogtle L, Rowe J, Barman J. The Pediatric Motor Activity Log-Revised: assessing real-world arm use in children with cerebral palsy. Rehabil Psychol. 2012;57(2):149-158.

[15]. World Health Organization. International Classification of Functioning, Disability and Health: ICF. Geneva: WHO, 2001.

[16]. Penta M, Tesio L, Arnould C, Zancan A, Thonnard J. The ABILHAND questionnaire as a measure of manual ability in chronic stroke patients. Raschbased validation and relationship to upper limb impairment. Stroke, 2001;32:1627-1634.

[17]. Morris D \& Taub E. Constraint-induced therapy approach to restoring function after neurological injury.. Topics in Stroke Rehabilitation, 2001;8(3):1630.
[18]. Sakzewski L, Ziviani J and Boyd R. Efficacy of upper limb therapies for unilateral cerebral palsy: a metaanalysis. Pediatrics 2014;133(1):e175-204.

[19]. Ziviani J, Poulsen A, Hansen C. Movement skills proficiency and physical activity: a case for Engaging and Coaching for Health (EACH)-Child. Australian Occupational Therapy Journal 2009;56(4):259-65.

[20]. Sakzewski L., Ziviani J., Fabott D., Macdonnell A., Graeme J., \& Boyd R. Randomized trial of constraintinduced movement therapy and bimanual training on activity outcomes for children with congenital hemiplegia. Developmental Medicine and Child Neurology, 2011;5:313-320.

[21]. Charles J, Gordon A. A critical review of constraintinduced movement therapy and forced use in children with hemiplegia. Neural Plast 2005;12:24561.

[22]. Lin K, Wang T., Wu C., Chen C., Chang K., Lin Y., \& Chen $Y$. Effects of home-based constraint-induced therapy versus dose-matched control intervention on functional outcomes and caregiver well-being in children with cerebral palsy. Research in developmental disabilities, 2011;32(5):1483-1491.

[23]. Taub E, Griffin A, Nick J, Gammons K, Uswatte G, Law C. Pediatric $\mathrm{Cl}$ therapy for stroke-induced hemiparesis in young children. Dev Neurorehabil. 2007;10:318.

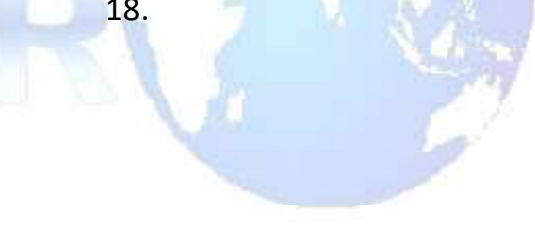

How to cite this article:

Asmaa Osama Sayed, Hebatallah Mohamed Kamal, Nagham Mohamed Kamal Khalil. CONSTRAINT INDUCED MOVEMENT THERAPY AND REALWORLD ARM USE IN CHILDREN WITH HEMIPARESIS. Int J Physiother Res 2017;5(3):2095-2100. DOI: 10.16965/ijpr.2017.149 